\title{
Integrity and Continuity of Sensor-Based Collision Warning Systems Using Vehicle-to-Vehicle Communication
}

\author{
Gustavo Lee \\ Aerospace and Mechanical \\ Department \\ The University of Arizona \\ Tucson, Arizona \\ gustavolee@email.arizona.edu
}

\author{
Mathieu Joerger \\ Aerospace and Mechanical \\ Department \\ The University of Arizona \\ Tucson, Arizona \\ joerger@email.arizona.edu
}

\begin{abstract}
This paper describes the design and implementation of a new safety risk evaluation method for a sensor-based automotive collision warning system using vehicle-to-vehicle (V2V) communication. The paper provides an overview of the $\mathrm{V} 2 \mathrm{~V}$ basic safety message (BSM) format and of surrogate measures of safety (SMS) used to parameterize a vehicle encounter. BSM and SMS are then employed to quantify risk of collision and risk of false alerts. Preliminary simulations illustrate the methodology in an example multi-sensor intersection movement assist system.
\end{abstract}

Keywords-integrity, collision warning, vehicle-to-vehicle, connected, automated.

\section{INTRODUCTION}

In this paper, we derive a methodology to evaluate safety risks in road vehicle encounters for a sensor-based collision warning system using vehicle-to-vehicle (V2V) communication. The call for certification and standardization in automated vehicle technology is becoming increasingly insistent [1]. In response, our approach uses two certifiable, sensor-independent safety performance metrics, 'integrity' and 'continuity', which have been implemented in aviation navigation applications with a proven safety record.

Automobile crashes remain one of the leading causes of death in the United States. According to the 2015 traffic safety facts of the National Highway Traffic Safety Administration (NHTSA), the number of traffic fatalities in 2015 was 35,092 and the number of injuries was 2.44 million [2]. The annual economic cost associated with these traffic crashes was estimated to be approximately $\$ 230$ billion, which was nearly $2.3 \%$ of the nation's gross domestic product [3].

The U.S. Department of Transportation (DOT) has estimated that V2V communication can address $82 \%$ of all crashes in the United States [4]. V2V communication is an automobile communication system that can be used to effectively recognize collision situations earlier and more accurately than human drivers. It relies on broadcast of position and velocity estimates (from GPS, inertial navigation systems or INS, and other sensors) between nearby vehicles to warn drivers about potential hazards. V2V communication systems can use dedicated short-range communication (DSRC), a technology designed to support a variety of applications for vehicular communication. DSRC is a two-way short to medium-range wireless communications capability that permits very high data transmission, which is key in road traffic applications [5]. As an alternative to DSRC, Long-Term Evolution (LTE) signals may be used, and could offer even greater capabilities [6].

The U.S. Federal Communications Commission (FCC) has allocated $75 \mathrm{MHz}$ of licensed spectrum in the $5.9 \mathrm{GHz}$ band for use by Intelligent Transportation Systems (ITS) vehicle safety and mobility applications [5]. In addition, in the Society of Automotive Engineers (SAE) J2735 Standard, the DSRC committee specifies a set of messages and their formats to support vehicle-based applications [7]. Of particular relevance to this work is the basic safety message (BSM), which conveys critical vehicle state information that includes vehicle position, positional accuracy, speed, heading, braking status, and size.

$\mathrm{V} 2 \mathrm{~V}$ communications utilizing DSRC have an operational range of about 300 meters [4]. Within this range, V2V applications have the potential to significantly reduce occurrences of crashes through real time advisories, alerting drivers to imminent hazards. GPS and GPS/INS-based relative positioning using V2V is subject to alteration and loss of GPS signal. But unlike vehicle-resident sensors (e.g., cameras and LiDARs), GPS/INS/V2V is not impacted by weather, light, or dust, and can sense out-of-sight vehicles occluded behind other vehicles or around building corners. This capability addresses scenarios where an oncoming vehicle emerges from behind a truck or from a blind alley. In those situations, GPS/INS /V2V can sense threats that a radar or camera cannot.

There are three collision scenarios that NHTSA believes can be mitigated by $\mathrm{V} 2 \mathrm{~V}$, and could not be addressed by any current, vehicle-resident sensor or camera-based system [4]. The first concept is the intersection movement assist (IMA). IMA warns the driver of a vehicle when it is not safe to enter an intersection due to the high probability of colliding with one 
or more vehicles at both controlled intersections (where a signal is present) and uncontrolled intersections (where only a stop or yield sign is present). The second concept is the left turn assist (LTA). LTA warns the driver of a vehicle, when entering an intersection, not to turn left in front of another vehicle traveling in the opposite direction. This is especially critical when the driver's line-of-sight is blocked by a vehicle also making a left turn from the opposite direction. The third concept is the emergency electronic brake light (EEBL). EEBL enables a vehicle to warn its driver to brake in a situation where another V2V-equipped vehicle decelerates quickly but may not be directly in front of the warning vehicle. V2V would allow the driver to "see through" vehicles or poor weather conditions to know that traffic ahead may be coming to an abrupt stop.

NHTSA indicates that the first two applications alone could result in a $50 \%$ reduction in crashes, injuries, and fatalities every year. Applied to the full national vehicle fleet for scenarios that would benefit from IMA and LTA, this could potentially eliminate 400,000 to 600,000 crashes, prevent 190,000 to 270,000 injuries and save 780 to 1,080 lives each year once fully deployed [8]. This has motivated several studies on collision alert using V2V [9-12].

This paper describes a new method to ensure the safety of GPS/INS/V2V collision warning systems by quantifying integrity and continuity. Integrity and continuity are performance metrics used in commercial aviation navigation. Integrity is a measure of trust in sensor information [15]. Continuity is the ability to complete a mission without interruption and is impacted by false alerts [15]. This paper uses analytical integrity and continuity evaluation methods to derive collision sensor requirements, thereby providing safe and timely collision warning alerts.

The next section of this paper presents the basic safety message (BSM) and its content. The third section describes the surrogate measures of safety. The fourth section outlines additional trajectory parameters needed to model a vehicle's encounter. We consider the example application of IMA, where two vehicles are approaching an intersection along perpendicular directions. The fifth section describes integrity and continuity risks. The integrity risk is defined as the probability of hazardously misleading information (HMI), whereas the continuity risk is defined as the probability of false alerts (FAs). Performance is evaluated in the sixth section by covariance analysis for example sensor specifications and safety requirements found in recent publications. At this stage of the research, we assume a preliminary constant-velocity vehicle encounter model. The method presented in this work provides the means to set collision system requirements to meet alert thresholds while ensuring pre-defined integrity and continuity risk requirements.

\section{BASIC SAFETY MeSSAGE}

V2V implementations are built around the SAE International J2735 Surface Vehicle Standard [7]. SAE J2735 currently specifies 15 message types. The Basic Safety Message (BSM) is the most relevant for safety-critical functions. It conveys state information about the broadcasting vehicle, namely its position, speed, heading, acceleration, and brake system status. The BSM has two parts. Part I includes critical state information that must be sent in every BSM. The data structure for Part I emphasizes compactness and efficiency, and contains 39 bytes. Part II is an optional area where additional data elements and frames can be included. It allows for the inclusion of some data types at a frequency less than the overall BSM rate based upon events (e.g. ABS activated), as well as the evolution in the definition of new state information and new applications [7]. The BSM is tailored for low latency, localized broadcast required by the V2V safety applications. The contents of the BSM are listed in Table 1.

In this paper, we will use the contents of the BSM as main sources of information for the analysis. This includes vehicle position, positioning accuracy, speed, and size. The SAE J2735 standard on BSM does not include any information on the variance of measured vehicle speed, but SAE is currently developing the SAE J2945 Minimum Performance Requirements to specify data accuracy. Since measured speed variance is a key parameter in our proposed method, we have included it in our analysis.

TABLE I. OVERview of Basic SAFEty Message Contents [7]

\begin{tabular}{|c|c|}
\hline Item name and length & Description \\
\hline $\begin{array}{l}\text { DSRC_MessageID } \\
1 \text { byte }\end{array}$ & $\begin{array}{l}\text { The first element in every message, used by } \\
\text { the parser to determine how to parse the rest } \\
\text { of the message. }\end{array}$ \\
\hline $\begin{array}{l}\text { MsgCount } \\
1 \text { byte }\end{array}$ & $\begin{array}{l}\text { A sequence number, incremented with each } \\
\text { successive transmission, primarily used to } \\
\text { estimate packet error statistics. }\end{array}$ \\
\hline $\begin{array}{l}\text { TemporaryID } \\
4 \text { bytes }\end{array}$ & $\begin{array}{l}\text { A value chosen randomly and held constant } \\
\text { for a few minutes to help the receiver } \\
\text { correlate a stream from a given sender. }\end{array}$ \\
\hline $\begin{array}{l}\text { DSecond } \\
2 \text { bytes }\end{array}$ & Current time. \\
\hline $\begin{array}{l}\text { Latitude, Longitude } \\
4 \text { bytes each }\end{array}$ & Geographic latitude and longitude. \\
\hline $\begin{array}{l}\text { Elevation } \\
2 \text { bytes }\end{array}$ & Position above or below sea level. \\
\hline $\begin{array}{l}\text { PositionalAccuracy } \\
4 \text { bytes }\end{array}$ & $\begin{array}{l}\text { Conveys the one-standard-deviation } \\
\text { position error along both semi-major and } \\
\text { semi-minor axes, and the heading of the } \\
\text { semi-major axis. }\end{array}$ \\
\hline $\begin{array}{l}\text { TransmissionAndSpeed } \\
2 \text { bytes }\end{array}$ & $\begin{array}{l}3 \text { bits encode vehicle transmission (gear), } \\
13 \text { bits convey unsigned vehicle speed. }\end{array}$ \\
\hline $\begin{array}{l}\text { Heading } \\
2 \text { bytes }\end{array}$ & Compass heading of vehicle's motion. \\
\hline $\begin{array}{l}\text { SteeringWheelAngle } \\
1 \text { byte }\end{array}$ & Current position of the steering wheel. \\
\hline $\begin{array}{l}\text { AccelerationSet } 4 \text { Way } \\
7 \text { bytes }\end{array}$ & $\begin{array}{l}\text { Provides longitudinal acceleration, lateral } \\
\text { acceleration, vertical acceleration, and yaw } \\
\text { rate. }\end{array}$ \\
\hline $\begin{array}{l}\text { BrakeSystemStatus } \\
2 \text { bytes }\end{array}$ & $\begin{array}{l}\text { Conveys whether or not braking is active on } \\
\text { each of the four wheels, also conveys the } \\
\text { status of the following control systems: } \\
\text { Traction Control, Anti-Lock Brakes, } \\
\text { Stability Control, Brake Boost, and } \\
\text { Auxiliary Brakes. }\end{array}$ \\
\hline $\begin{array}{l}\text { VehicleSize } \\
3 \text { bytes }\end{array}$ & Vehicle length and width \\
\hline
\end{tabular}




\section{Surrogate MEASURES OF SAFETy}

The safety of traffic operations is typically measured by the number of traffic accidents. However, this number can be unreliable because data is scarce (the number of incidents at intersections of interest can be statistically insignificant). For these reasons, alternative approaches in traffic safety evaluation have been pursued.

In 1967, General Motors (GM) developed the Traffic Conflicts Technique (TCT) to test how GM compared with other auto-makers in terms of car safety [16]. The procedure involved systematically observing or qualifying evasive actions such as sudden lane-changing or hard braking as a clue to deduce critical situations [3]. According to TCT, such measurement of accident potential is a suitable surrogate measure for accidents, and can be used as an effective and reliable safety metric in the evaluation of traffic operations, without the need for the accumulation of historical accident data.

TCT captured the attention of traffic safety researchers and initiated multiple studies in the U.S. and overseas. The early studies of traffic conflicts revealed the inherent inadequacies of the original definition of conflicts, which led into several alternate methods oriented toward more objective measurements of conflicts [16]. These methods have ultimately resulted in using surrogate measures, a concept adopted from the medical industry, to measure safety. Tarko, Saunier, and Sayed state that for a surrogate measure of safety (SMS) to be useful for transportation safety applications, it should be based on "an observable non-crash event that is physically related in a predictable and reliable way to crashes" [17]. Over time, the definition of a traffic conflict has been refined and an internationally accepted definition of a traffic conflict has become "an observable situation in which two or more road users approach each other in space and time for such an extent that there is a risk of collision if their movements remain unchanged." [18,19].

In 1978, Allen, Shin, and Cooper introduced several SMS to define a traffic conflict [20,31]:

- Gap time (GT): Time lapse between completion of encroachment by the offending (turning) vehicle and the arrival time of the crossing vehicle if they continue with the same speed and path.

- Encroachment Time (ET): Time duration which the turning vehicle infringes upon the right-of-way of the through vehicle.

- Deceleration Rate (DR): Rate at which the crossing vehicle must decelerate to avoid a collision.

- Proportion of Stopping Distance (PSD): Ratio of distance available to maneuver to the distance remaining to the projected location of collision.

- Post-Encroachment Time (PET): Time lapse between end of encroachment of turning vehicle and the time that the through vehicle actually arrives at the potential point of collision.
- Initially Attempted Post-Encroachment Time (IAPT): Time lapse between commencement of encroachment by turning vehicle plus the expected time for the through vehicle to reach the point of collision and the completion time of encroachment by turning vehicle.

- Time to Collision (TTC): Expected time for two vehicles to collide if they remain at their present speed and on the same path.

The most widely used surrogate measure is the time to collision (TTC) defined as "the time for two vehicles to collide if they continue at their present speed and on the same path" [21]. As defined, TTC is infinite if the vehicles are not on a collision course. If the vehicles are on a collision course (i.e., they arrive at the conflict point at or near the same time), the value of the TTC is finite and decreases with both time and/or speed. Driver responses can prevent collisions, i.e., prevent TTC from reaching zero, or uncomfortably small values.

Time-To-Collision has proven to be an effective measure for rating the severity of traffic conflicts. Results of several studies point to the direct use of TTC as a cue for decisionmaking in traffic [22]. For this reason, TTC will be used in the methodology proposed in this paper. An important issue in developing Collision Warning Systems (CWS) is defining the proper warning strategy that warns the driver only when the driver is really at danger and immediate action is required, while minimizing the rate of false alarms (FA) that can become a nuisance to the driver. In the next sections, we provide an analytical evaluation method that takes into account both sides of the equation to create a warning strategy provides safe collision warning alerts, while also minimizing the FA rate.

\section{INTERSECTION MOVEMENT ASSIST}

In this paper, we consider the Intersection Movement Assist (IMA) scenario, one of the applications the NHTSA indicates has the potential to significantly reduce crashes, injuries, and fatalities. In the IMA scenario, the system warns the driver if it is not safe to enter an intersection.

Figure 1 illustrates the intersection and the associated parameters that we will use to define our scenario. The vehicle traveling northward (up) is the host vehicle and the vehicle traveling westward (right to left) is the intruder vehicle. The space in which the vehicles' respective lanes intersect is the Common Spatial Zone (CSZ). The CSZ is defined as a square area of side length $H$, the width of the road. The remaining variables are defined as follows:

$d_{h} \quad:$ is the host vehicle's distance to CSZ

$d_{i} \quad:$ is the intruder vehicle's distance to CSZ

$v_{h}, v_{i}$ : respectively are the host and intruder vehicle speeds

$L_{h}, L_{i} \quad$ : respectively are the host and intruder vehicle lengths

The variables $d_{h}, d_{i}, v_{h}, v_{i}$ are the trajectory states. 


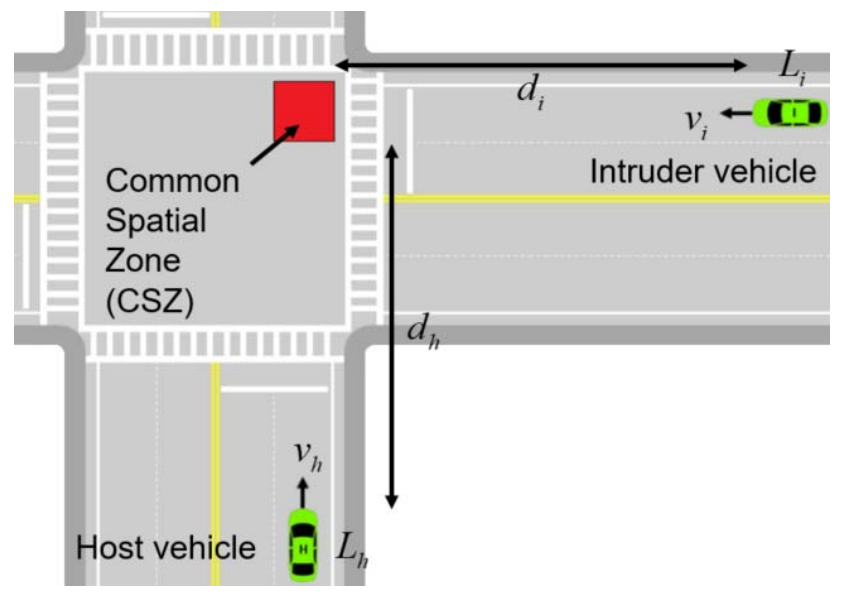

Fig. 1. Illustration of the Intersection Movement Assist Scenario

For the problem illustrated in Figure 1, there are two ways to define TTC. First, if the host vehicle arrives at the CSZ first, the TTC is defined in terms of the intruder vehicle's position and speed. Conversely, if the intruder vehicle arrives at the CSZ first, the TTC is defined in terms of the host vehicle's position and speed. In both instances, the approaching vehicles must be on a collision-course for TTC to be defined. In other words, if the host vehicle arrives at the CSZ first, a conflict (potential collision) is defined only if the intruder vehicle arrives before the host vehicle's full-length has cleared the CSZ. Otherwise, the vehicles are merely on a crossing-course, and not a collision-course [23]. In these cases, TTC is infinite. The three cases can be summarized as follows:

$$
\begin{gathered}
\tau_{T T C}=\frac{d_{i}}{v_{i}} \text { if } \frac{d_{h}}{v_{h}} \leq \frac{d_{i}}{v_{i}} \leq \frac{d_{h}+H+L_{h}}{v_{h}} \\
\tau_{T T C}=\frac{d_{h}}{v_{h}} \text { if } \frac{d_{i}}{v_{i}} \leq \frac{d_{h}}{v_{h}} \leq \frac{d_{i}+H+L_{i}}{v_{i}} \\
\tau_{T T C}=\infty, \text { otherwise }
\end{gathered}
$$

\section{INTEGRITY AND CONTINUITY RISK}

\section{A. Integrity Risk}

In aviation, integrity is defined as the trust that can be placed in the correctness of information supplied by the system [15]. Integrity risk is quantified as the probability that the collision warning system provides hazardously misleading information (HMI), which is an unacceptably large error without a timely warning that the system cannot be trusted [24]. In this problem, HMI occurs if the collision warning system does not sense a hazard, when one exists:

$$
P_{H M I}=P(\text { Sense No Hazard } \mid \text { Hazard Exists })
$$

Using the definition of TTC, a hazard exists if the TTC is below a pre-defined threshold, $T_{T T C}$, set by the certification authority (e.g. NHTSA). To differentiate risky encounters from situations in which the driver remains safely in control, an appropriate threshold or critical value $T_{T T C}$ must be defined. Generally, TTC is related to the perception and reaction time of the driver. However, in order to properly define the $T_{\text {TTC }}$ threshold, the vehicle's deceleration capabilities must also be taken into account. Thus, the TTC threshold must allow the driver sufficient time to perceive, process, and react to the hazard, in addition to providing the vehicle sufficient time to stop before colliding into the hazard, given a reasonable deceleration rate. This is known as the stopping sight distance, converted to a time based on the vehicle's speed [25,33]. Section VI of this paper provides references and a rationale for setting $T_{T T C}$.

Using the definition of TTC given in (1-3), a hazard is defined if the following event occurs:

$$
\begin{aligned}
\text { Hazard Exists } \equiv & {\left[\tau_{h} \leq \tau_{i} \cap \tau_{i} \leq T_{T T C} \cap d_{E, h} \leq T_{E, h}\right] } \\
& \cup\left[\tau_{i} \leq \tau_{h} \cap \tau_{h} \leq T_{T T C} \cap d_{E, i} \leq T_{E, i}\right]
\end{aligned}
$$

where:

$\tau_{h}, \tau_{i}:$ are the times for the host and intruder vehicles to reach the CSZ, respectively

$d_{E, h}, d_{E, i}$ : refer to the total encroachment distance for the host and intruder, respectively

$T_{E, h} \quad:$ is the encroachment distance threshold when the host reaches CSZ first: $T_{E, h} \equiv H+L_{h}$

$T_{E, i} \quad:$ is the encroachment distance threshold when the intruder reaches CSZ first: $T_{E, i} \equiv H+L_{i}$

The variables $T_{E, h}$ and $T_{E, i}$ are the distances the encroaching vehicle needs to clear before the other vehicle arrives. If the encroachment distance ( $d_{E, h}$ or $d_{E, i}$ ) is less than the width of the road $(H)$ plus the length of the encroaching vehicle $(L)$, then there will be a collision when the second vehicle arrives. Mathematically, $d_{E, h}$ and $d_{E, i}$ are described by the following equations:

$$
\begin{gathered}
d_{E, h} \equiv\left(v_{h} \tau_{i}-d_{h}\right) \\
d_{E, i} \equiv\left(v_{i} \tau_{h}-d_{i}\right)
\end{gathered}
$$

Given that a hazard exists when the event in (5) occurs, the collision warning system does not sense a hazard if the following is true:

Sense No Hazard $\equiv\left(\begin{array}{c}{\left[\begin{array}{c}\left.\hat{\tau}_{h} \leq \hat{\tau}_{i} \cap\left(\hat{\tau}_{i}>T_{T T C} \cup \hat{d}_{E, h}>T_{E, h}\right)\right] \\ \cup\left[\hat{\tau}_{i} \leq \hat{\tau}_{h} \cap\left(\hat{\tau}_{h}>T_{T T C} \cup \hat{d}_{E, i}>T_{E, i}\right)\right.\end{array}\right]}\end{array}\right)$ 
where the 'hat' symbols indicate estimates of the corresponding variables, e.g., $\hat{\tau}_{h}$ is an estimate of $\tau_{h}$.

The four variables $\hat{\tau}_{h}, \hat{\tau}_{i}, \hat{d}_{E, h}$, and $\hat{d}_{E, i}$ are estimates of the hazard states, which are functions of the trajectory states we defined in Section IV. The trajectory states are obtained from GPS/INS and speedometer sensor measurements as explained in Appendix A. We use overbounding theory to determine the uncertainty in $\hat{\tau}_{h}, \hat{\tau}_{i}, \hat{d}_{E, h}$, and $\hat{d}_{E, i}$ caused by measurement errors. Assuming the measurement errors can be overbounded in their cumulative distribution functions (CDFs) by Gaussian distributions, the hazard state estimates can also be overbounded by Gaussian distributions [13,14,26]. Under this assumption, the probability distributions of estimation errors in $\hat{\tau}_{h}, \hat{\tau}_{i}, \hat{d}_{E, h}$, and $\hat{d}_{E, i}$ are known.

For risk evaluation purposes, we can simplify the expression in (8) by considering the following union of events, which includes the event in (8):

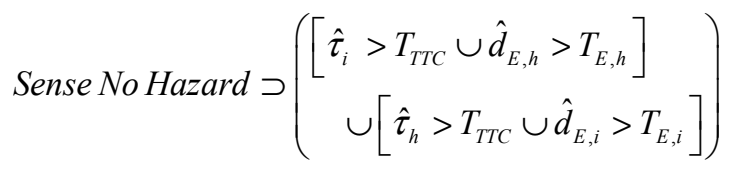

The integrity risk, or probability of HMI, $P_{H M I}$, is defined as:

$$
P_{H M I} \leq P(\text { Sense No Hazard } \mid \text { Hazard Exists })
$$

with the inequality used because of the simplified expression in (9) that ensures a bound on $P_{H M I}$. Substituting (9) into (10), we can express a bound on $P_{H M I}$ as:

$$
P_{H M I} \leq\left(\begin{array}{l}
P\left(\hat{\tau}_{i}>T_{T T C} \mid \text { Hazard Exists }\right) \\
+P\left(\hat{d}_{E, h}>T_{E, h} \mid \text { Hazard Exists }\right) \\
+P\left(\hat{\tau}_{h}>T_{T T C} \mid \text { Hazard Exists }\right) \\
+P\left(\hat{d}_{E, i}>T_{E, i} \mid \text { Hazard Exists }\right)
\end{array}\right)
$$

The bound in (11) remains valid even though the hazard state estimates $\hat{\tau}_{h}, \hat{\tau}_{i}, \hat{d}_{E, h}$, and $\hat{d}_{E, i}$ are correlated. It is conservative because, for example, when evaluating $\left[\hat{\tau}_{h}>T_{T T C} \cup \hat{d}_{E, i}>T_{E, i}\right]$, the intersection of events is counted twice, once in $P\left(\hat{\tau}_{h}>T_{T T C}\right)$, and once in $P\left(\hat{d}_{E, i}>T_{E, i}\right)$.

$P_{H M I}$ must meet a predefined integrity risk requirement $I_{R E Q}$, which is defined as a function of the host and intruder vehicles' relative velocity as described in Appendix B. This is expressed as:

$$
P_{H M I} \leq I_{R E Q}
$$

In order to meet this requirement, we can adjust the hazard state thresholds by a multiple of the hazard state estimate error standard deviations. By increasing the TTC threshold, we can ensure that alerts are sent early enough. By increasing the encroachment distance threshold, we can ensure, with quantifiable probability, that one vehicle has cleared the CSZ when the other reaches it. The following inequality must be met:

$$
\left(\begin{array}{l}
P\left(\hat{\tau}_{i}>T_{T T C}+k_{i} \sigma_{i} \mid \text { Hazard Exists }\right) \\
+P\left(\hat{d}_{E, h}>T_{E, h}+k_{E, h} \sigma_{E, h} \mid \text { Hazard Exists }\right) \\
+P\left(\hat{\tau}_{h}>T_{T T C}+k_{h} \sigma_{h} \mid \text { Hazard Exists }\right) \\
+P\left(\hat{d}_{E, i}>T_{E, i}+k_{E, i} \sigma_{E, i} \mid \text { Hazard Exists }\right)
\end{array}\right) \leq I_{R E Q}
$$

where:

$\sigma_{h}, \sigma_{i}$ :respectively are the hazard state estimate error standard deviations on $\hat{\tau}_{h}$ and $\hat{\tau}_{i}$

$\sigma_{E, h}, \sigma_{E, i}$ :respectively are the hazard state estimate error standard deviations on $\hat{d}_{E, h}$ and $\hat{d}_{E, i}$

and where $k_{h}, k_{i}, k_{E . h}$, and $k_{E, i}$, ae integrity multipliers derived below.

The question becomes: how much should we increase the hazard state thresholds? How large should the ' $k \sigma$ ' terms be? In order to ensure that (13) is satisfied, we consider the 'worstcase' condition of Hazard Exists in (5). We want the worst case in the sense that it maximizes $P_{H M I}$ because we want to upper-bound $P_{H M I}$. An optimistic lower-bound would not be safe. The Hazard Exists condition is expressed in terms of hazard states $\tau_{h}, \tau_{i}, d_{E, h}$, and $d_{E, i}$, which, under the Gaussian bounding assumption, correspond to the means of the hazard state estimates $\hat{\tau}_{h}, \hat{\tau}_{i}, \hat{d}_{E, h}$, and $\hat{d}_{E, i}$. At the limit of the 'Hazard Exists' condition, the hazard states reach their respective thresholds. At this point, the mean values of $\hat{\tau}_{h}, \hat{\tau}_{i}$, $\hat{d}_{E, h}$, and $\hat{d}_{E, i}$ are maximized, which maximizes $P_{H M I}$. Thus, the worst case is: $\tau_{h}=T_{T T C}, \tau_{i}=T_{T T C}, d_{E, h}=T_{E, h}$, and $d_{E, i}=T_{E, i}$. Equation (13) can be rewritten as:

$$
\left(\begin{array}{l}
P\left(\hat{\tau}_{i}>T_{T T C}+k_{i} \sigma_{i} \mid \tau_{i}=T_{T T C}\right) \\
+P\left(\hat{d}_{E, h}>T_{E, h}+k_{E, h} \sigma_{E, h} \mid d_{E, h}=T_{E, h}\right) \\
+P\left(\hat{\tau}_{h}>T_{T T C}+k_{h} \sigma_{h} \mid \tau_{h}=T_{T T C}\right) \\
+P\left(\hat{d}_{E, i}>T_{E, i}+k_{E, i} \sigma_{E, i} \mid d_{E, i}=T_{E, i}\right)
\end{array}\right) \leq I_{R E Q}
$$


In order to satisfy (14), it is sufficient to allocate the integrity risk requirement $I_{R E Q}$ evenly across the four terms in the left-hand-side sum of probabilities. Therefore, we can write:

$$
\begin{aligned}
& P\left(\hat{\tau}_{i}>T_{T T C}+k_{i} \sigma_{i} \mid \tau_{i}=T_{T T C}\right) \leq I_{R E Q} / 4 \\
& P\left(\hat{d}_{E, h}>T_{E, h}+k_{E, h} \sigma_{E, h} \mid d_{E, h}=T_{E, h}\right) \leq I_{R E Q} / 4 \\
& P\left(\hat{\tau}_{h}>T_{T T C}+k_{h} \sigma_{h} \mid \tau_{h}=T_{T T C}\right) \leq I_{R E Q} / 4 \\
& P\left(\hat{d}_{E, i}>T_{E, i}+k_{E, i} \sigma_{E, i} \mid d_{E, i}=T_{E, i}\right) \leq I_{R E Q} / 4
\end{aligned}
$$

Figure 2 provides a 2D illustration of the $P_{H M I}$-bounding process based on $\hat{\tau}_{h}$ and $\hat{d}_{E, i}$. The concentric ellipses represent lines of constant joint probability, with tilted major axes because the random variables $\hat{\tau}_{h}$ and $\hat{d}_{E, i}$ are correlated. They are centered at the threshold intersection, because this is the worst case described in (14).

In this example, the vertical shaded area where $\hat{\tau}_{h}>T_{\text {TTC }}+k_{h} \sigma_{h}$ shows the region where collision warning system believes that the host vehicle has sufficient time to prevent a collision. The probability of being in the horizontal shaded area where $\hat{d}_{E, i}>T_{E, i}+k_{E, i} \sigma_{E, i}$ represents the risk of the host vehicle sensing that the encroaching intruder vehicle has cleared the common spatial zone. The union of these two areas is $P_{H M I}$. The expression provided in (11) double counts the upper right quadrant, thus providing an upper bound on $P_{H M I}$. A graphical representation for a similar approach to deal with state estimate correlation is provided in [27,28] for Unmanned Aircraft System (UAS) Sense and Avoid (SAA) missions.

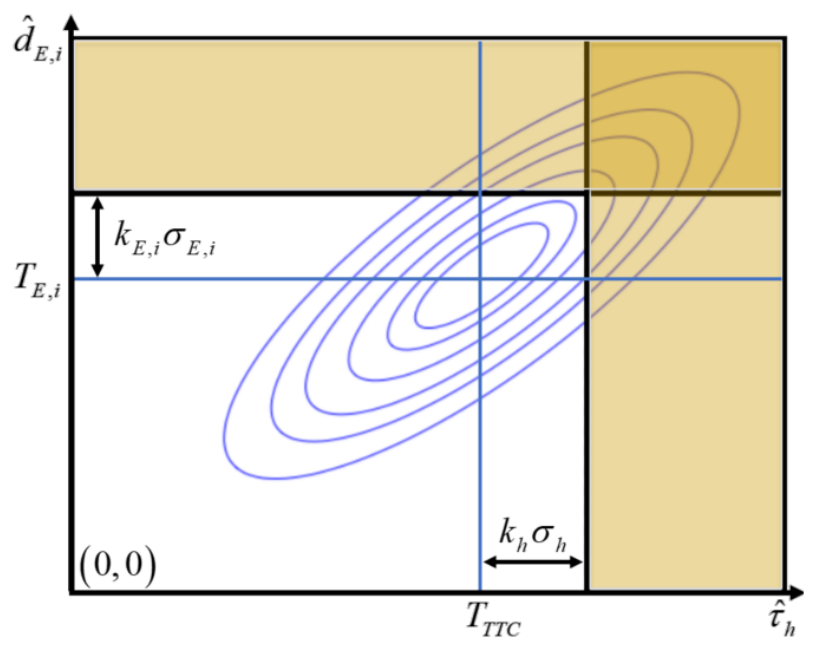

Fig. 2. Graphical Representation of Conservative Bound on $P_{H M I}$
To obtain closed-form expressions for the multipliers $k_{h}, k_{i}, k_{E, h}$, and $k_{E, i}$, we consider the equivalent inequalities:

$$
\begin{aligned}
& P\left(\hat{\varepsilon}_{i}>k_{i} \sigma_{i}\right) \leq I_{R E Q} / 4 \\
& P\left(\hat{\varepsilon}_{E, h}>k_{E, h} \sigma_{E, h}\right) \leq I_{R E Q} / 4 \\
& P\left(\hat{\varepsilon}_{h}>k_{h} \sigma_{h}\right) \leq I_{R E Q} / 4 \\
& P\left(\hat{\varepsilon}_{E, i}>k_{E, i} \sigma_{E, i}\right) \leq I_{R E Q} / 4
\end{aligned}
$$

where we defined: $\hat{\varepsilon}_{i} \equiv \hat{\tau}_{i}-\tau_{i}$, and under the corresponding condition in (15), we have: $\hat{\varepsilon}_{i} \equiv \hat{\tau}_{i}-T_{T T C}$. The hazard state estimation error $\hat{\varepsilon}_{i}$ is normally distributed with zero mean and variance $\sigma_{i}^{2}$. We use the notation: $\hat{\varepsilon}_{i} \sim N\left(0, \sigma_{i}^{2}\right)$. Similarly, we define $\hat{\varepsilon}_{h}, \hat{\varepsilon}_{E, h}, \hat{\varepsilon}_{i}$, and $\hat{\varepsilon}_{E, i}$ as:

$$
\begin{aligned}
& \hat{\varepsilon}_{h} \equiv \hat{\tau}_{h}-\tau_{h}=\hat{\tau}_{h}-T_{T T C} \sim N\left(0, \sigma_{h}^{2}\right) \\
& \hat{\varepsilon}_{E, h} \equiv \hat{d}_{E, h}-d_{E, h}=\hat{d}_{E, h}-T_{E, h} \sim N\left(0, \sigma_{E, h}^{2}\right) \\
& \hat{\varepsilon}_{i} \equiv \hat{\tau}_{i}-\tau_{i}=\hat{\tau}_{i}-T_{T T C} \sim N\left(0, \sigma_{i}^{2}\right) \\
& \hat{\varepsilon}_{E, i} \equiv \hat{d}_{E, i}-d_{E, i}=\hat{d}_{E, i}-T_{E, i} \sim N\left(0, \sigma_{E, i}^{2}\right)
\end{aligned}
$$

The integrity multipliers can then be derived from (16) using the following equations:

$$
Q\left(k_{h}\right)=Q\left(k_{i}\right)=Q\left(k_{E, h}\right)=Q\left(k_{E, i}\right)=I_{R E Q} / 4
$$

where $Q()$ is the tail probability of the standard normal distribution with zero mean and unit variance:

$$
Q(x)=\frac{1}{2}\left[1-\operatorname{erf}\left(\frac{x}{\sqrt{2}}\right)\right]
$$

An equivalent expression for the multipliers is given by:

$$
k_{h}=k_{i}=k_{E, h}=k_{E, i}=Q^{-1}\left(I_{R E Q} / 4\right)
$$

where $Q^{-1}()$ is the inverse tail probability distribution.

In summary, we have derived a conservative, easy-toimplement method to guarantee an upper-bound on $P_{H M I}$. The adjusted thresholds of the form ' $T+k \sigma$ ' in (15) guarantee that $I_{R E Q}$ is achieved. These thresholds can be implemented in simulations to derive sensor requirements, and can be implemented in operation to determine the last time instance when it is still safe to send an alert to the driver. Beyond that point, automated braking may be required. 


\section{B. Continuity Risk}

Continuity can be defined as the ability of the total system to perform its function without unscheduled interruptions [15]. The major contributor to continuity risk is the probability of false (or early) alerts. A false alert (FA) can be defined as the probability of the system sensing a hazard when a hazard does not exist:

$$
P_{F A}=P(\text { Sense Hazard } \mid \text { No Hazard Exists })
$$
if:

Using a similar formulation as HMI, a hazard does not exist

$$
\begin{aligned}
\text { No Hazard Exists } & \equiv\left[\tau_{h} \leq \tau_{i} \cap\left(\tau_{i}>F_{T T C} \cup d_{E, h}>T_{E, h}\right)\right] \\
& \cup\left[\tau_{i} \leq \tau_{h} \cap\left(\tau_{h}>F_{T T C} \cup d_{E, i}>T_{E, i}\right)\right]
\end{aligned}
$$

where $F_{\text {TTC }}$ is the TTC non-hazard threshold set by the certification authority (NHTSA). The non-hazard threshold is defined as the point away from the conflict zone beyond which an alert does not contribute to the safety of the vehicle/driver, but acts as a nuisance. Alerts must not be late, but they should not be too early either, or there is a risk of traffic disturbance. $F_{\text {TTC }}$ is defined based on driver reaction time, and on a minimum deceleration rate ( $F_{\text {TTC }}$ setting is further discussed in Section VI).

The collision warning system may start sending alerts if it is in a hazard zone, i.e., out of the no-hazard zone. This can be expressed in terms of hazard state estimates as:



We again simplify this expression by considering the following union of events, which includes the event in (23):

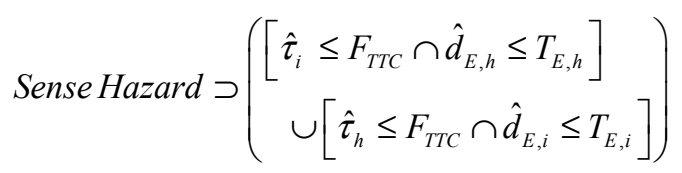

Following a procedure similar to the one in the previous section for HMI, we can express a bound on $P_{F A}$ as:

$$
P_{F A} \leq\left(\begin{array}{c}
\frac{P\left(\hat{\tau}_{i} \leq F_{T T C} \mid N H E\right)+P\left(\hat{d}_{E, h} \leq T_{E, h} \mid N H E\right)}{2} \\
+\frac{P\left(\hat{\tau}_{h} \leq F_{T T C} \mid N H E\right)+P\left(\hat{d}_{E, i} \leq T_{E, i} \mid N H E\right)}{2}
\end{array}\right)
$$

where NHE $\equiv$ No Hazard Exists . The probability of the intersection of events $\left[\hat{\tau}_{i} \leq F_{T T C} \cap \hat{d}_{E, h} \leq T_{E, h}\right]$ in (24) is counted twice in $P\left(\hat{\tau}_{i} \leq F_{T T C} \mid N H E\right)+P\left(\hat{d}_{E, h} \leq T_{E, h} \mid N H E\right)$, hence the factor $1 / 2$ in (25). An excess probability causes the bound to be conservative, but will provide practical closed form expressions in $P_{F A}$ evaluation. The excess probability caused by the first two terms of the sum of four probabilities in (25) is:

$$
\frac{1}{2}\left[\begin{array}{r}
P\left(\hat{\tau}_{i} \leq F_{T T C} \mid N H E\right)+P\left(\hat{d}_{E, h} \leq T_{E, h} \mid N H E\right) \\
-P\left(\hat{\tau}_{i} \leq F_{T T C} \cap \hat{d}_{E, h} \leq T_{E, h} \mid N H E\right)
\end{array}\right]
$$

Then, adjusting the hazard thresholds by a multiple of the hazard state estimate error standard deviations, and allocating the continuity risk requirement $C_{R E Q}$ equally to the four terms on the right-hand-side of (25), the continuity risk criterion is given by the following inequalities:

$$
\begin{aligned}
& P\left(\hat{\tau}_{i} \leq F_{T T C}+l_{i} \sigma_{i} \mid \tau_{i}=F_{T T C}\right) \leq C_{R E Q} / 2 \\
& P\left(\hat{d}_{E, h} \leq T_{E, h}+l_{E, h} \sigma_{E, h} \mid d_{E, h}=T_{E, h}\right) \leq C_{R E Q} / 2 \\
& P\left(\hat{\tau}_{h} \leq F_{T T C}+l_{h} \sigma_{h} \mid \tau_{h}=F_{T T C}\right) \leq C_{R E Q} / 2 \\
& P\left(\hat{d}_{E, i} \leq T_{E, i}+l_{E, i} \sigma_{E, i} \mid d_{E, i}=T_{E, i}\right) \leq C_{R E Q} / 2
\end{aligned}
$$

Finally, using this even allocation, the four continuity risk multipliers are given by:

$$
l_{h}=l_{i}=l_{E, h}=l_{E, i}=Q^{-1}\left(C_{R E Q} / 2\right)
$$

These continuity risk multipliers can be used to determine an early alert boundary, which takes into account measurement errors, and within which the $P_{F A}$ requirement $\left(C_{R E Q}\right)$ is satisfied.

Similar to Figure 2, we use a simple 2D example to illustrate the expression given in (25), as shown in Figure 3. In this example, the vertical shaded area where $\hat{\tau}_{h} \leq F_{T T C}+l_{h} \sigma_{h}$ shows the region where collision warning system believes that it is time for the host vehicle to send an alert. The horizontal shaded area where $\hat{d}_{E, i} \leq T_{E, i}+l_{E, i} \sigma_{E, i}$ represents the region where the encroaching vehicle (in this case, the intruder) has not yet cleared the common spatial zone. The intersection of these two areas is $P_{F A}$. The expression provided in (25) accounts for excess probability in addition to the intersecting area, thus providing an upper bound on $P_{F A}$. 




Fig. 3. Graphical Representation of Conservative Bound on $P_{F A}$

In summary, our proposed method defines a safe alert zone between the early alert threshold $\left(F_{T T C}+l_{i} \sigma_{i}\right)$ and the late alert threshold $\left(T_{T T C}+k_{i} \sigma_{i}\right)$. It is in this zone that the collision warning system can issue a warning to the vehicle that satisfies both the integrity risk and continuity risk requirements.

\section{PERFORMANCE ANALYSIS FOR AN EXAMPLE APPLICATION}

This analysis assumes a two-dimensional, constant-velocity scenario. The initial conditions are as follows:

$$
\begin{array}{ll}
v_{h}=20 \mathrm{~m} / \mathrm{s} & (45 \text { miles per hour }) \\
v_{i}=25 \mathrm{~m} / \mathrm{s} \quad(56 \text { miles per hour })
\end{array}
$$

The integrity risk requirement is defined as in Appendix B. It is a function of the relative velocity, which in this case, relates to an integrity risk requirement of $10^{-9}$. The continuity risk requirement is set to $10^{-5}$. The speed measurement uncertainty is set to $\sigma_{v}=0.1 \mathrm{~m} / \mathrm{s}$ based on an assumed speedometer uncertainty, and the position measurement uncertainty is varied from 0 to $20 \mathrm{~m}$. The early and late alert thresholds are calculated using driver reaction times and vehicle deceleration times. Four recent studies have shown maximum perception-reaction times of 1.9 seconds for an $85^{\text {th }}$ percentile time [32]. The early alert threshold is found by using a deceleration rate of $0.34 \mathrm{~g}$ 's (the nominal deceleration level described in [25]) and a reaction time of 2 seconds. The late alert threshold, on the other hand, is calculated using a deceleration rate of $0.68 \mathrm{~g}$ 's (defined as emergency braking in [32] or twice the nominal value) and a reaction time of 1 second. The results are shown in Figure 4.

Figure 4 shows the early alert and late alert thresholds in blue and red, respectively. It also shows the adjusted thresholds for the hazard state $\tau_{h}$. As we vary the GPS position measurement uncertainty, the adjusted thresholds of the form
' $T+k \sigma$ ' from (14) and (27), converge until they overlap. For this particular example, the maximum GPS position measurement uncertainty that the collision warning system can tolerate is about $4.7 \mathrm{~m}$. At this critical value, the alert zone disappears and the collision warning system is not able to issue an alert to the vehicle/driver without violating either the integrity or the continuity risk requirements.

Similarly, the sensitivity on speed measurement uncertainty was analyzed. The position measurement uncertainty is set to $\sigma_{p}=1 \mathrm{~m}$ and the speed measurement uncertainty is varied from 0 to $2 \mathrm{~m} / \mathrm{s}$, Figure 5 shows a similar plot for speed. For this particular example, the maximum speed measurement uncertainty that the collision warning system can tolerate is about $0.56 \mathrm{~m} / \mathrm{s}$. At this critical value, the alert zone disappears and the collision warning system is not able to issue an alert to the vehicle/driver without violating either the integrity or the continuity risk requirements.

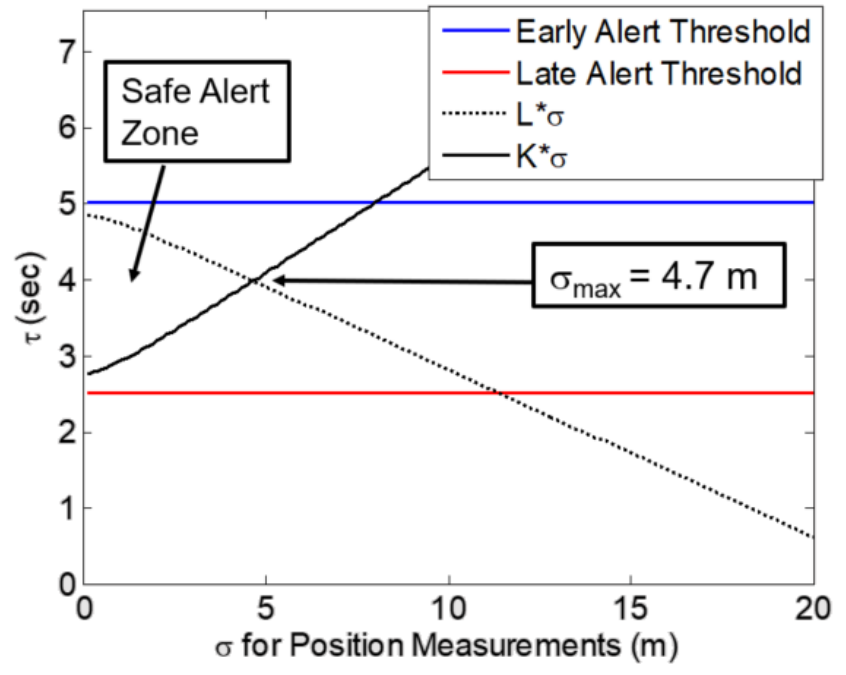

Fig. 4. Safe Alert Zone vs. GPS Position Uncertainty

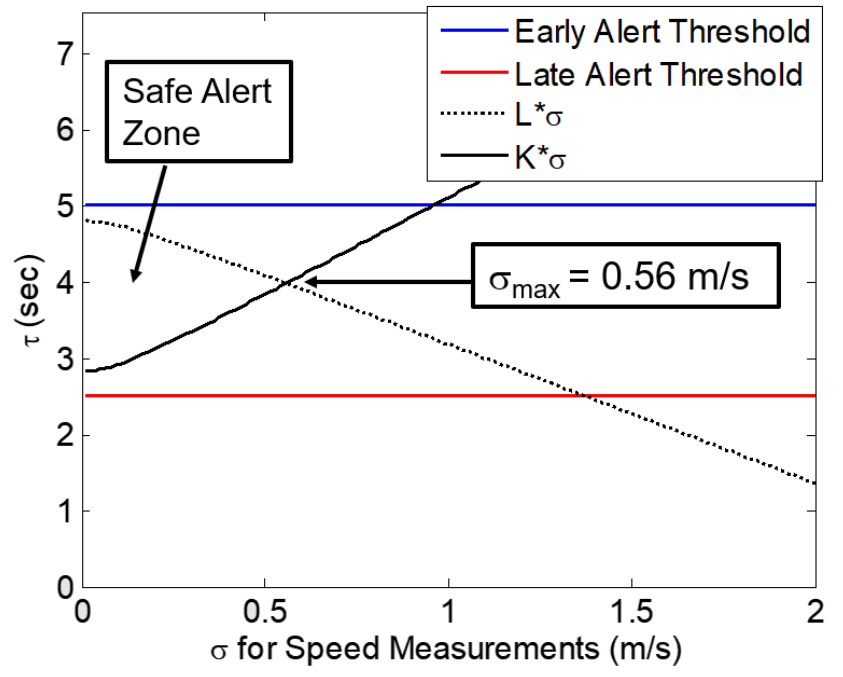

Fig. 5. Safe Alert Zone vs. Speedometer Uncertainty 


\section{CONCLUSION}

Vehicle-to-vehicle communication (V2V) technology can be used to increase the safety of road vehicles and reduce collisions and injuries. In this paper, we derived and evaluated a new method for determining safety-based early and late alert thresholds for a V2V collision warning system in Intersection Movement Assist (IMA). The method was implemented in an example simulation to set sensor requirements, which ensured that pre-defined integrity and continuity risk specifications could be achieved.

\section{APPENDIX A: HAZARD STATES}

The hazard states $\tau_{h}, \tau_{i}, d_{E, h}, d_{E, i}$ can be derived from the trajectory states as follows:

$$
\begin{gathered}
\tau_{h}=\frac{d_{h}}{v_{h}} \\
\tau_{i}=\frac{d_{i}}{v_{i}} \\
d_{E, h} \equiv\left(v_{h} \tau_{i}-d_{h}\right) \\
d_{E, i} \equiv\left(v_{i} \tau_{h}-d_{i}\right)
\end{gathered}
$$

To get the hazard state estimates $\hat{\mathbf{x}}_{N}$ and its covariance matrix $\hat{\mathbf{P}}_{N}$, a weighted least squares estimator is used:

$$
\begin{gathered}
\hat{x}_{n}=\left(H_{N}^{T} V_{N}^{-1} H_{N}\right)^{-1} H_{N}^{T} V_{N}^{-1} z_{N} \\
\hat{P}_{N}=\left(H_{N}^{T} V_{N}^{-1} H_{N}\right)^{-1}
\end{gathered}
$$

where our measurement vector $\mathbf{z}_{N}$ consists of GPS-based position measurements and speedometer-based velocity measurements. Our batch observation matrix and batch measurement error covariance matrix will be of the following form:

$$
\begin{gathered}
H_{N}=\left[\begin{array}{cc}
I_{3 \times 3} & 0_{3 \times 3} \\
\vdots & \vdots \\
I_{3 \times 3} & n \Delta t I_{3 \times 3}
\end{array}\right] \\
V_{N}=\left[\begin{array}{ccc}
V_{0} & \ldots & 0 \\
\vdots & \ddots & \vdots \\
0 & \cdots & V_{n}
\end{array}\right]
\end{gathered}
$$

To get the hazard state estimate error variances, we linearize them using a first-order Taylor series expansion in terms of the trajectory states, for example:

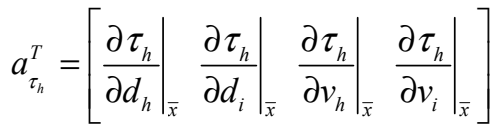

where:

\section{$\bar{x}$ :is the a priori estimate of the trajectory states}

The $\tau_{h}$ estimate error variance $\sigma_{h}^{2}$ can be determined using the following equation:

$$
\sigma_{h}^{2}=a_{\tau_{h}}^{T} \hat{P}_{N} a_{\tau_{h}}
$$

\section{APPENDIX B: INTEGRITY RISK REQUIREMENT}

In $[29,30]$, the relationship between vehicle speed and injury severity is analyzed for seat-belted car drivers in collisions with other cars. The data shows that for frontal impacts $50 \%$ of slight injuries occur at $15 \mathrm{mph}, 50 \%$ of serious injuries occur at $25 \mathrm{mph}$, and $50 \%$ of fatal injuries occur at 35 mph. For side impacts, the numbers are the following: $50 \%$ of slight injuries occur at $7 \mathrm{mph}, 50 \%$ of serious injuries occur at $15 \mathrm{mph}$, and $50 \%$ of fatal injuries occur at $25 \mathrm{mph}$.

In parallel, reference [29] classifies system failures (minor, major, catastrophic) in terms of their effects on vehicle occupants (discomfort, injuries, fatalities), and provides corresponding risk requirements $\left(10^{-3}\right.$ to $10^{-5}, 10^{-5}$ to $10^{-7}, 10^{-7}$ to $\left.10^{-9}\right)$. Using this information, we defined preliminary integrity requirements, which varies with relative vehicle speed, as shown in Figure 6.



Fig. 6. Example Integrity Risk Requirement as a Function of Vehicle Relative Speed

\section{REFERENCES}

[1] D. Bevly, X. Cao, M. Gordon, G. Ozbilgin, D. Kari, B. Nelson, J. Woodruff, M. Barth, C. Murray, A. Kurt, K. Redmill, and U. Ozguner, "Lane Change and Merge Maneuvers for Connected and Automated Vehicles: A Survey," IEEE Trans on Intelligent Vehicles, Vol. 1, No. 1, 2016

[2] National Highway Traffic Safety Administration, "Traffic Safety Facts: 2015 Motor Vehicle Crashes: Overview," August 2016.

[3] H. Yang. Simulation-based evaluation of traffic safety performance using surrogate safety measures. Ph.D. dissertation, Rutgers, New Brunswick, NJ, 2012.

[4] J. Harding et al., "Vehicle-to-vehicle communications: Readiness of V2V technology for application. (Report No. DOT HS 812 014).," 
Washington, DC: National Highway Traffic Safety Administration, August 2014.

[5] J. B. Kenney, "Dedicated short-range communications (DSRC) Standards in the United States," Proceedings of the IEEE, vol. 99, no. 7, July 2011.

[6] Qualcomm, “Accelerationg C-V2X commercialization,” 2017.

[7] Dedicated Short Range Communications (DSRC) Message Set Dictionary, SAE Std. J2735, SAE Int., DSRC Committee, Nov. 2009.

[8] "Vehicle-to-Vehicle Communication Technology Fact Sheet," U.S. Department of Transportation.

[9] S.B. Raut, P.R. Bajaj, and L.G. Malik, "Prediction of Vehicle Collision Probablity at Intersection using V2V Communication," International Journal of Scientific \& Engineering Research, Volume 6, Issue 5, 2015

[10] R. Dang, J. Ding, B. Su, Q. Yao, Y. Tian, and K. Li, "A Lane Change Warning System Based on V2V Communication," Proc. IEEE 17th International Conference on Intelligent Transportation Systems (ITSC), Qingdao, China, 2014.

[11] C.M. Huang, and S. Lin, "An Early Collision Warning Algorithm for Vehicles Based on V2V Communication," International Journal of Communication Systems, 2012.

[12] L.N. Chen, L. Liu, Q. Pei, and X. Li, "Critical Safe Distance Design to Improve Driving Safety Based on Vehicle-to-Vehicle Communications," Journal of Intelligent Transportation System, 2012.

[13] J. Rife, S. Pullen, P. Enge, and B. Pervan, "Paired overbounding for nonideal LAAS and WAAS error distributions," IEEE Transactions on Aerospace and Electronic Systems, vol. 42, no. 4, pp. 1386-1395, October 2006.

[14] J. Rife, S. Pullen, B. Pervan, and P. Enge, "Paired overbounding and application to GPS augmentation," Position Location and Navigation Symposium, pp. 439-446, 2004.

[15] R. J. Kelly and J. M. Davis, "Required Navigation Performance (RNP) for Precision Approach and Landing with GNSS Application," Navigation: Journal of the Institute of Navigation, vol. 41, no. 1, pp. 130, 1994.

[16] M. Ghaffari, A stochastic computer simulation of traffic conflicts. Ph.D. dissertation, University of Illinois, Chicago, IL, 1990.

[17] A. Tarko, N. Saunier, and T. Sayed, "Surrogate Mesaures of Safety," White Paper, April, 2009.

[18] T. Sayed and S. Zein, "Traffic conflict standards for intersections," Transportation Planning and Technology, vol. 22, pp. 309-323, March 1998.

[19] F. Amundsen, and C. Hyden. "Proc., First Workshop on Traffic Conflicts.” Institute of Transport Economics, Oslo, Norway, 1977.
[20] B. L. Allen, B. T. Shin, and P. J. Cooper, "Analysis of traffic conflicts and collisions." In Transportation Research Record 667, TRB, National Research Council, Washington, D.C., 1978, pp. 67-74.

[21] J. Hayward, "Near miss determination through use of a scale of danger." Report TTSC-7115, The Pennsylvania State University, University Park, 1972

[22] R. van der Horst and J. Hogema, "Time-to-collision and collision avoidance systems," Proc., Sixth ICTCT Workshop Salzburg. TNO Institute for Human Factors, The Netherlands, 1994.

[23] A. Laureshyn, A. Svensson, and C. Hyden, "Evaluation of traffic safety, based on micro-level behavioural data: Theoretical framework and first implementation," Accident Analysis and Prevention, vol. 42, pp. 16371646, March 2010.

[24] RTCA Special Committee 159, "Minimum Aviation System Performance Standards for the Local Area Augmentation System (LAAS)," RTCA/DO-245, 2004, Appendix D.

[25] "A Policy on Geometric Design of Highways and Streets," American Association of State Highway and Transportation Officials (AASHTO), Washington D.C., 2011.

[26] B. DeCleene, "Defining pseudorange integrity - overbounding," Proceedings of the Institute of Navigation GPS Conference, The Institute of Navigation (ION), Manassas, VA, Sept. 2000, pp. 19161924.

[27] M. B. Jamoom, M. Joerger, and B. Pervan, "Unmanned Aircraft System Sense-and-Avoid Integrity and Continuity Risk," Journal of Guidance, Control, and Dynamics, vol. 39, no. 3, pp. 498-509, March 2016.

[28] M. B. Jamoom, Unmanned aircraft system sense and avoid integrity and continuity, Ph.D. dissertation, Illinois Institute of Technology, Chicago, IL, 2016.

[29] D. Richards and R. Cuerden, "The relationship between speed and car driver injury severity," Transport Research Laboratory, London, April 2009.

[30] D. C. Richards, "Relationship between speed and risk of fatal injury: pedestrians and car occupants," Transport Research Laboratory, London, September 2010.

[31] D. Gettman and L. Head, "Surrogate safety measures from traffic simulation models, Final report (Report No. FHWA-RD-03-050)," Federal Highway Administration.

[32] Federal Highway Administration, "Vehicle positioning trade study for ITS applications (Report No. FHWA-JPO-12-064),” April 2012.

[33] R. Layton, "Decision Sight Distance," The Kiewit Center for Infrastructure and Transportation, March 2012. 